\title{
Microscopic Mechanism of the Helix-to-Layer Transformation in Elemental Group VI Solids
}

\author{
Dan Liu, ${ }^{\dagger}$ Xianqing Lin, ${ }^{\dagger, \downarrow}$ and David Tománek ${ }^{*, \dagger}$ \\ $\dagger$ †hysics and Astronomy Department, Michigan State University, East Lansing, Michigan \\ 48824, USA \\ $\ddagger$ College of Science, Zhejiang University of Technology, Hangzhou 310023, China \\ E-mail: tomanek@pa.msu.edu
}

\begin{abstract}
We study the conversion of bulk Se and Te, consisting of intertwined $a$ helices, to structurally very dissimilar, atomically thin twodimensional (2D) layers of these elements. Our $a b$ initio calculations reveal that previously unknown and unusually stable $\delta$ and $\eta$ 2D allotropes may form in an intriguing multi-step process that involves a concerted motion of many atoms at dislocation defects. We identify such a complex reaction path involving zipperlike motion of such dislocations that initiate structural changes. With low activation barriers $\lesssim 0.3 \mathrm{eV}$ along the optimum path, the conversion process may occur at moderate temperatures. We find all one-dimensional (1D) and $2 \mathrm{D}$ chalcogen structures to be semiconducting.
\end{abstract}

\section{Keywords}

microscopic conversion mechanism, ab initio calculation, elemental semiconductor, electronic structure

After much attention has been devoted to graphene, a 2D allotrope of group IV elemental carbon, scientific interest turned to semiconducting 2D allotropes of group $\mathrm{V}$ elements $\mathrm{P}^{1,2}$ and As. ${ }^{3,4}$ Recent observation of $2 \mathrm{D}$ allotropes of group VI elements Se and $\mathrm{Te}^{5-7}$ came as a surprise, since - unlike group IV and V elemental solids - the bulk structure of Se and Te is not layered, but consists of helical chains of covalently bonded atoms packed in a hexagonal array. For lack of well-defined layers, 2D Se and Te can not be obtained by mechanical exfoliation used in group IV and V systems. Chalcogens are known for a large number of stable allotropes and oxidation states. ${ }^{8}$ The latter fact had been identified as the key factor behind the stability of specific 2D allotropes of Se and Te. ${ }^{5}$ Still, the strong dissimilarity between the bulk structure containing weakly interacting, intertwined $a$ helices and covalently bonded, atomically thin layers raises the intriguing question about the microscopic mechanism behind the transformation from quasi-1D to 2D structures, which has not been addressed yet.

Here we present results of $a b$ initio calculations, which help to identify the intermediate steps of the observed transition from $a$ helices in the native bulk structure to atomically thin layers of elemental Se and Te. ${ }^{5}$ Our results unveil the energetics and the intermediate steps encountered during this structural transition. We have discovered an intriguing mechanism that converts an $a$ helix to a more stable, previously unknown $b$ chain by moving a pointdislocation connecting these two structures. In a zipper-like motion, the $b$ chain may reconnect to a previously unknown $2 \mathrm{D} \delta$ structure, which is unusually stable, similar to the related $\eta$ structure. The structural change from the $a$ helix to the $2 \mathrm{D} \delta$ allotrope is mildly exothermic with $-0.17 \mathrm{eV} /$ atom for Se and $-0.23 \mathrm{eV} /$ atom 


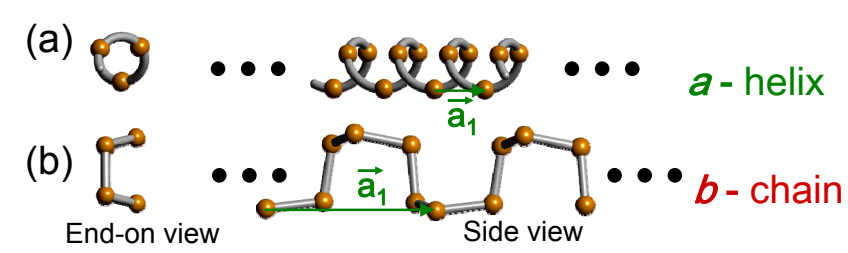

(c)
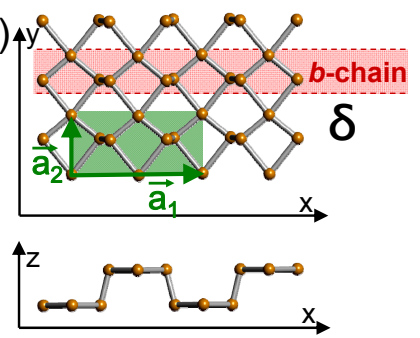

(d)

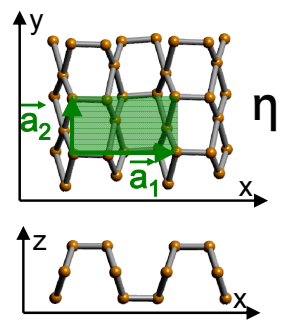

Figure 1: (Color online) Stable 1D and 2D structural allotropes of Se and Te. 1D structures of the (a) $a$ helix and (b) $b$ chain and their 2D counterparts, the (c) $\delta$ and (d) $\eta$ allotrope. The $\delta$ allotrope is a covalently bonded $2 \mathrm{D}$ assembly of $b$ chains. The unit cells of the 2D structures are highlighted by the transparent green areas in (c) and (d).

for Te. The low number of structural constraints allows the helical structure to exploit many degrees of freedom and thus to lower the activation barriers along the reaction path to $\lesssim 0.3 \mathrm{eV}$, indicating that the transition may occur at moderate temperatures. Our GW quasiparticle calculations of the electronic structure indicate that all quasi-1D and 2D chalcogen allotropes are semiconducting.

\section{Results}

\section{Formation of 2D monolayers of Se and Te from 1D a helices}

Understanding the observed $1 \mathrm{D}$ to $2 \mathrm{D}$ transformation is an unprecedented challenge due to the large and constantly changing number of degrees of freedom that are actively involved in lowering the activation barriers between intermediate states. In this complex system, the use of common techniques such as the nudged elastic band model becomes a futile endeavor. Restricting the system's freedom invariably increases the activation barriers, incorrectly suggesting that the transformation should not oc-
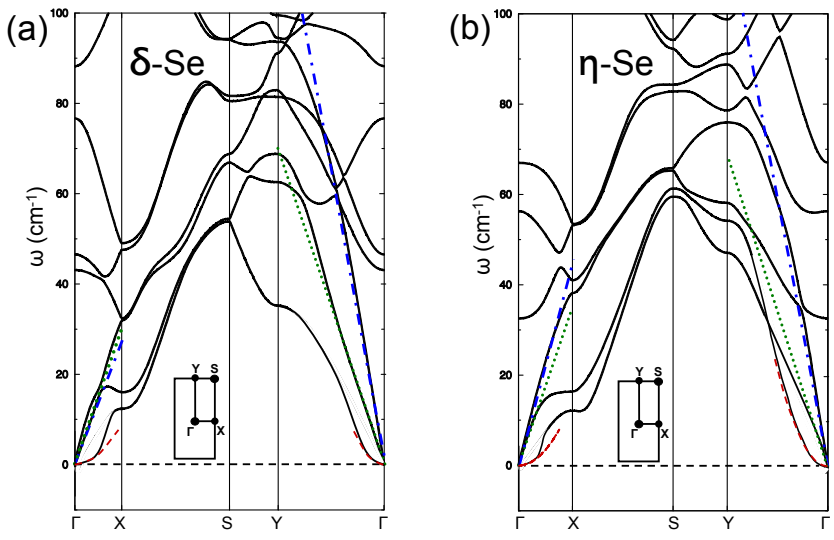

Figure 2: (Color online) Phonon spectra of (a) $\delta$-Se and (b) $\eta$-Se calculated using the DFTLDA energy functional. The Brillouin zones and high-symmetry points are shown schematically in the insets. Continuum elasticity results for long-wavelength longitudinal acoustic modes are shown by the blue dash-dotted lines, for transverse acoustic modes by the dotted green lines, and for flexural modes by the dashed red parabolas.

cur under laboratory conditions. We chose a different approach that will be discussed in the following.

Recently observed 2D Se and Te structures $^{5-7,9}$ have been formed by initially evaporating the bulk substances. It is likely that the vapor contained primarily short segments of $a$ helices, shown in Fig. 1(a), which constitute the bulk structure. Consequently, we will consider the $a$ helix as the initial structure in the transformation process to $2 \mathrm{D}$ structures. We discovered a previously unknown, atomically thin and unusually stable 2D structure of Se and Te, which we call the $\delta$ structure, by artificially compressing a 2D assembly of the native $a$-Se helices in the direction normal to the 2D layer. The $2 \mathrm{D} \delta$ allotrope, shown in Fig. 1(c), emerged after the pressure was released. The specific conditions for this deformation process are specified in the Supporting Information. Another previously unknown and stable allotrope, labeled $\eta$, is depicted in Fig. $1(\mathrm{~d})$. It is related to the $\delta$ structure by a series of reflections, discussed in the Supporting Information, while keeping the bond lengths and bond angles constant throughout the struc- 

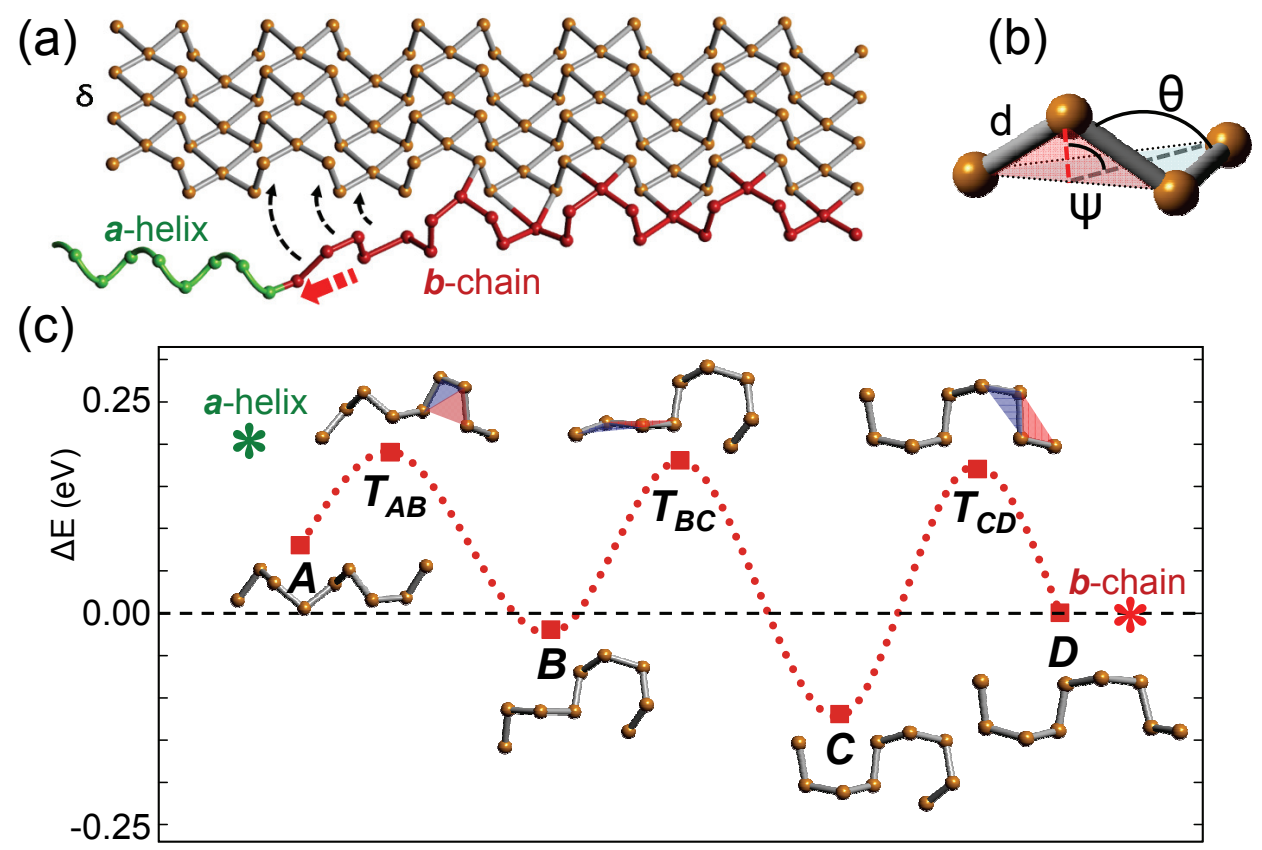

Reaction Coordinate

Figure 3: (Color online) (a) Schematic growth mechanism of the energetically stable 2D $\delta$ structure by zipper-like attachment of the $b$ chain, which is being formed locally locally at a defect in the native $a$ helix and propagates by dislocation motion. (b) Bond length $d$, bond angle $\theta$ and dihedral angle $\psi$ used to characterize chalcogen structures. (c) DFT-LDA based energy differences $\Delta E$ encountered during the stepwise conversion from 1D $a$-Se to $b$-Se as a function of the reaction coordinate. The system is represented by a finite $\mathrm{Se}_{9} \mathrm{H}_{2}$ chain, passivated by hydrogen at both ends, and the total energy is given with respect to the final state. The dotted line is guide to the eye. The energy of a 9-atom long segment of the defect-free infinite $a$-Se and $b$-Se chains is indicated by asterisks. Ball-and-stick models show stable $\mathrm{Se}_{9} \mathrm{H}_{2}$ geometries, labeled by $A$ - $D$, and the transition states $T$. Location of the unusually small dihedral angle in the transition states is indicated by shaded triangles.

ture. The space group of the $\delta$ structure is $C_{2 v}^{4}$ in the Schönflies notation and its group number is \#28. The space group of the $\eta$ structure is $D_{2}^{2}$ in the Schönflies notation and its group number is \#17. Both groups have only 4 symmetry operations. Numerical results for the cohesive energies of all known Se and Te allotropes are summarized in Table 1.

We studied the stability of the new phases by determining their elastic response and their phonon spectra. Since the 3D elastic modulus tensor is not defined for a truly 2D system, which does not naturally form layered 3D compounds, we have determined the components of the 2D elastic tensor defined earlier. ${ }^{10}$ For the $\delta$-phase of Se, we find $c_{11}=$ $4.97 \mathrm{~N} / \mathrm{m}, c_{22}=20.02 \mathrm{~N} / \mathrm{m}, c_{66}=5.92 \mathrm{~N} / \mathrm{m}$, $D(\Gamma-X)=0.33 \mathrm{eV}, D(\Gamma-Y)=1.15 \mathrm{eV}$.
For the $\eta$-phase of Se, we find $c_{11}=11.25 \mathrm{~N} / \mathrm{m}$, $c_{22}=22.71 \mathrm{~N} / \mathrm{m}, c_{66}=7.09 \mathrm{~N} / \mathrm{m}, D(\Gamma-X)=$ $0.39 \mathrm{eV}, D(\Gamma-Y)=1.02 \mathrm{eV}$. Among others, these elastic constants allow a more accurate representation of low-frequency acoustic modes in the vibrational band structure of the $2 \mathrm{D}$ structures, which we present in Fig. 2. Due to the similarity of the phonon spectra, we expect the zero-point motion to not to play an important role in the cohesive energy. We find a zero-point energy of $23 \mathrm{meV} /$ atom for $\beta$-Se and $24 \mathrm{meV} /$ atom for $\delta$-Se and $\eta$-Se, with energy differences of $\lesssim 0.1 \mathrm{meV} /$ atom between the different phases.

We have also confirmed the dynamic stability of the $1 \mathrm{D}$ and $2 \mathrm{D}$ structures by performing canonical molecular dynamics (MD) simulations at elevated temperatures. Results of 4 ps 
Table 1: Cohesive energy $E_{c o h}$ of various Se and Te allotropes in [eV/atom] units, obtained using DFT-LDA and DFT-PBE calculations.

\begin{tabular}{ccccccccc}
\hline \hline & & $a$-helix & $b$-chain & $\alpha$ & $\beta$ & $\gamma$ & $\delta$ & $\eta$ \\
\hline \multirow{2}{*}{ Se } & LDA & 3.677 & 3.700 & 3.795 & 3.823 & 3.569 & 3.843 & 3.854 \\
& PBE & 3.307 & 3.327 & 3.198 & 3.302 & 2.953 & 3.357 & 3.355 \\
\hline \multirow{2}{*}{ Te } & LDA & 3.209 & 3.235 & 3.479 & 3.434 & 3.323 & 3.443 & 3.451 \\
& PBE & 2.856 & 2.871 & 2.904 & 2.933 & 2.716 & 2.940 & 2.943 \\
\hline \hline
\end{tabular}

long runs for the $b$ chain at $300 \mathrm{~K}$ and $500 \mathrm{~K}$, and of a 2 ps run for $\delta$-Se at $300 \mathrm{~K}$ are shown as videos in the Supporting Information.

In the following, we will first address structures of elemental Se and refer discussion of Te structures for later. Our DFT-LDA and DFTPBE results suggest that $\delta$-Se and $\eta$-Se are energetically near-degenerate. The previously introduced $^{5,6} \beta$-Se allotrope is less stable than $\delta$ Se by $20 \mathrm{meV} /$ atom (LDA) and $55 \mathrm{meV} /$ atom (PBE) and thus the least stable of the three. Still, in view of the relatively small energy differences and structural similarities, we expect that all these structural allotropes, and possibly even others, may be formed under synthesis conditions at elevated temperatures. In the following, we will focus on the energetically stable $\delta$ structure and its microscopic formation mechanism starting from the native $a$ helix structure.

Inspecting the equilibrium structure of $\delta$-Se in Fig. 1(c), we found that it can be viewed as a 2D assembly of $1 \mathrm{D}$ chains, which we call $b$ chains. We found the previously unknown $b$ chain, shown in Fig. 1(b), to be a stable allotrope of Se, even more stable than the 1D a helix by $23 \mathrm{meV} /$ atom (LDA) and $20 \mathrm{meV} /$ atom (PBE). The $b$ chain may be attached laterally to a semi-infinite $\delta$-Se layer in a zipper-like motion depicted in Fig. 3(a). Owing to the multi-valent behavior of the chalcogens, ${ }^{5}$ this is an activation-free exothermic process that releases $143 \mathrm{meV} /$ atom (LDA) and $30 \mathrm{meV} /$ atom (PBE).

Assuming that $b$-Se chains, which are entropically favored at high temperatures, are indeed present during the formation of $\delta$-Se, then the only task remaining to understand the entire conversion path from $a$-Se to $\delta$-Se is locating an energetically favorable pathway for the transformation from the $a$ helix to the $b$ chain. The most plausible transformation begins by connecting the semi-infinite $a$ helix and a $b$ chain end-to-end by a covalent bond, as seen in Fig. 3(a). The $a-b$ connection is a dislocation defect or a 0D domain wall that may propagate along the $1 \mathrm{D}$ chain, as indicated by the broken arrow in Fig. 3(a) and in a schematic movie in the Supporting Information. For Se, the step-wise $a$-to- $b$ conversion is exothermic and requires only a finite activation energy to be discussed below. Individual processes within the entire $a$-to- $b$-to- $\delta$ transformation may occur concurrently, as shown in Fig. 3(a).

We noted that observed stable allotropes of Se and Te all share structural commonalities. As defined in Fig. 3(b), these include the bond length $d(\mathrm{Se}) \approx 2.38 \AA$ and $d(\mathrm{Te}) \approx 2.84 \AA$, the bond angle $\theta \approx 100^{\circ}-130^{\circ}$, and the dihedral angle $\psi \approx 80^{\circ}-100^{\circ}$ found experimentally in bulk structures. ${ }^{11-15}$ The step-wise dislocation motion corresponds to a series of $a$-Se to $b$ Se structural changes at the dislocation, which were studied in finite chain segments containing 12 Se atoms, passivated by hydrogen at both ends. Relaxing the finite segment of $a$ Se yielded structure $A$ and relaxing the finite $b$-Se segment resulted in structure $D$. Interestingly, the infinite $a$ and $b$ chains as well as their finite counterparts $A$ and $D$ displayed very similar structural characteristics as the bulk structures. Later on, we found out that shorter, 9atom segments shown in Fig. 3(c), are sufficient to visualize and understand the step-wise transformations in the 1D structure.

We discovered that the relative stability of the infinite $a$-Se and $b$-Se chains, as well as that of the optimized finite segments, can be rationalized in terms of strain originating in the devia- 
tion from the optimum bond length $d=2.36 \AA$, bond angle $\theta \approx 106^{\circ}$ and dihedral angle $\psi \approx 83^{\circ}$, defined in Fig. 3(b). Most robust of these parameters is the bond length, which is close to its optimum value in all optimized structures. Whereas $\theta=102^{\circ}$ and $\psi=100^{\circ}$ are constant throughout the unit cell of $a$-Se, two thirds of the $b$-Se unit cell display $\theta=105^{\circ}$ and $\psi=83^{\circ}$, and the rest is characterized by $\theta=101^{\circ}$ and $\psi=100^{\circ}$. The closer proximity of $b$-Se to the optimum angles $\theta$ and $\psi$ is reflected in its higher stability by $23 \mathrm{meV} /$ atom (LDA) and $20 \mathrm{meV} /$ atom (PBE) with respect to $a$-Se.

We found that the entire $A$ to $D$ transformation can be accomplished by a sequence of bond rotations or reflection while maintaining the optimum bond lengths $d$ and bond angles $\theta$ throughout the structure. The transformation steps only involved changes in one dihedral angle $\psi$ at a time, as shown in Fig. 3(c), which required a typical activation energy of $\lesssim 300 \mathrm{meV}$. Our DFT-LDA energies for the process described in Fig. 3(c) differ from DFT-PBE results by $\lesssim 30 \mathrm{meV}$ and from van-der-Waals corrected DFT-optB86b results by $\lesssim 25 \mathrm{meV}$ for the entire structure. The similarity in cohesive energies obtained using LDA and PBE is also seen in Table 1 . We identified two locally stable structures, labeled $B$ and $C$, along the $A$ to $D$ trajectory. The locally stable states $A, B, C$ and $D$ all displayed near-optimum values of $d$, $\theta$ and $\psi$ throughout the structure. The contiguous trajectory in configurational space contains unstable transition states $T_{A B}$ between $A$ and $B, T_{B C}$ between $B$ and $C$, and $T_{C D}$ between $C$ and $D$. We traced back the lower stability of the transition states to one of the dihedral angles being near zero, far from its optimum value. We verified that all transition states $T$ were unstable in the sense that perturbing the $T_{N, N+1}$ structure in whichever way and following up with microcanonical MD calculations or conjugate gradient $(\mathrm{CG})$ optimization always lead to optimum $N$ or $N+1$ geometries and to no other structure. The relative energy and the structure of these states are depicted in Fig. 3(c). More details and MD simulations of the entire $A$ to $D$ transformation are presented in the Supporting Information. We expect the postulated transition process to be just one of many similar transformations in the system that may occur with potentially even lower activation barriers.

We should remember that the sequence and energetics of $A$ to $D$ transformations, identified in the finite chain segment, may differ in detail from the corresponding process at a dislocation defect connecting infinite $a$ and $b$ chains, since the free-standing finite structure has fewer constraints than the infinite structure. As seen in Fig. 3(c), the net energy gain from the infinite $a$ to the $b$ structure is higher than the energy gain in the finite segment changing from the $A$ to the $D$ structure. As a matter of fact, we should not place too much emphasis on the relative stability of finite $A, B, C$ and $D$ structures, but rather realize that the activation barriers for step-wise structural changes are similar in finite and infinite structures. The energetics and structure of the free-standing finite chain segment will change when connected to a semiinfinite $a$ chain at the one and a semi-infinite $b$ chain at the other end. Whatever differences in the relative stability of the intermediate states in the infinite or finite segment connecting $a$ and $b$ chains, none will stop the attachment of the $b$ chain to $\delta$-Se that occurs at a significant net energy gain of $143 \mathrm{eV} /$ atom, thus driving the exothermic reaction forward.

Since Te shares the same group VI with Se in the periodic table, we expect the chemical behavior and bonding in the respective elemental solids to be very similar. We found the formation mechanism of the $\delta$ allotrope from native $a$ helices via the $b$ chains, discussed above for Se, to be viable for Te as well, with small differences in reaction energies. According to Table 1 , the $a$-Te to $b$-Te conversion is exothermic, releasing $26 \mathrm{meV} /$ atom (LDA) and $15 \mathrm{meV} /$ atom(PBE). Attaching the $b$ chain laterally to a semi-infinite $\delta$-Te layer is also exothermic, releasing $208 \mathrm{meV} /$ atom (LDA) and $69 \mathrm{meV} /$ atom (PBE). As mentioned earlier, also the geometries of the initial, final and intermediate states encountered during the $a$ Te to $b$-Te transformation are similar, the main difference being the Te-Te bond length, which is larger than the value in Se allotropes. Most important, also activation energies of $\approx 0.3 \mathrm{eV}$ 

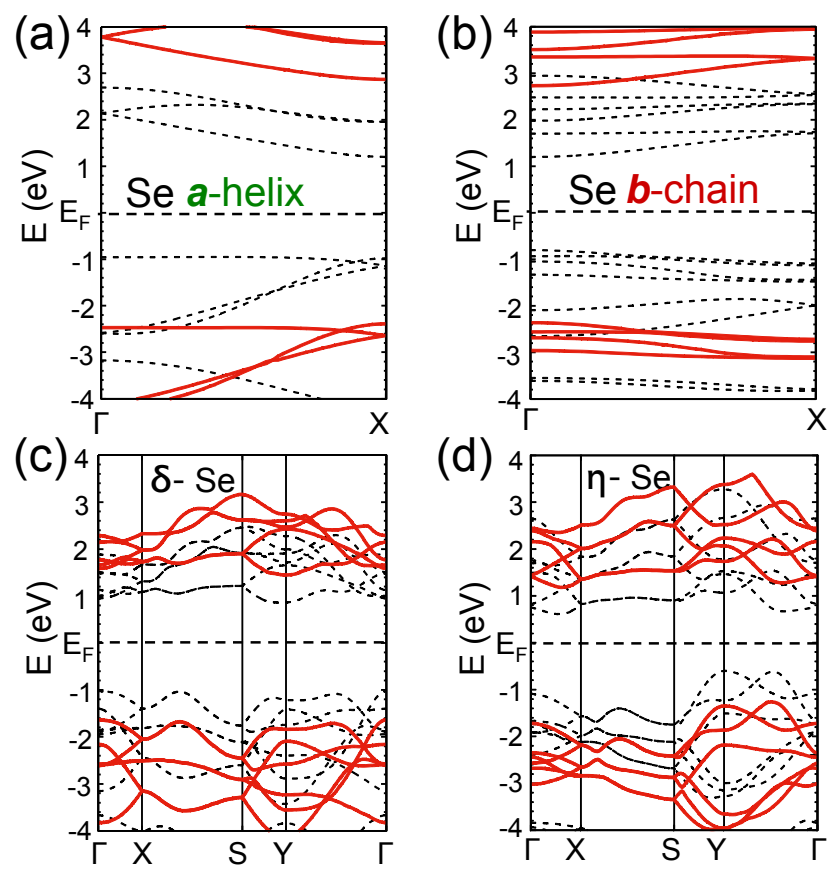

Figure 4: (Color online) Electronic band structure of an isolated (a) $a$-Se helix, (b) $b$-Se chain, isolated (c) $\delta$-Se and (d) $\eta$-Se monolayers. GW results, shown by solid red lines, are compared to LDA results, shown by the black dashed lines.

are similar in Te and Se.

\section{Electronic structure of $1 \mathrm{D}$ and $2 \mathrm{D}$ Se and Te allotropes}

The electronic band structure of the different 1D and 2D allotropes of Se is shown in Fig. 4. The GW results, shown by the solid red lines, are considered a valid counterpart to experimental observation. The LDA results, shown as a matter of reference by the dashed black lines, underestimate the fundamental band gap $E_{g}$ significantly. Among $1 \mathrm{D}$ structures, $a$-Se in Fig. 4(a) has an $E_{g}=5.3 \mathrm{eV}$ wide direct gap at $X$, and the $b$-Se in Fig. 4(b) has an $E_{g}=5.1 \mathrm{eV}$ wide direct gap at $\Gamma$. Our GW band gap for $a$-Se compares favorably with a recently reported $E_{g}=5.46 \mathrm{eV}$ value $^{16}$ and a smaller $E_{g}=3.00 \mathrm{eV}$ value ${ }^{17}$ obtained using a different approach. $\delta$-Se in Fig. 4(c) has an $E_{g}=3.1 \mathrm{eV}$ wide indirect gap, and $\eta$-Se in Fig. 4(d) has an $E_{g}=2.4 \mathrm{eV}$ wide indirect gap. In general, we see that the band gaps in $1 \mathrm{D}$
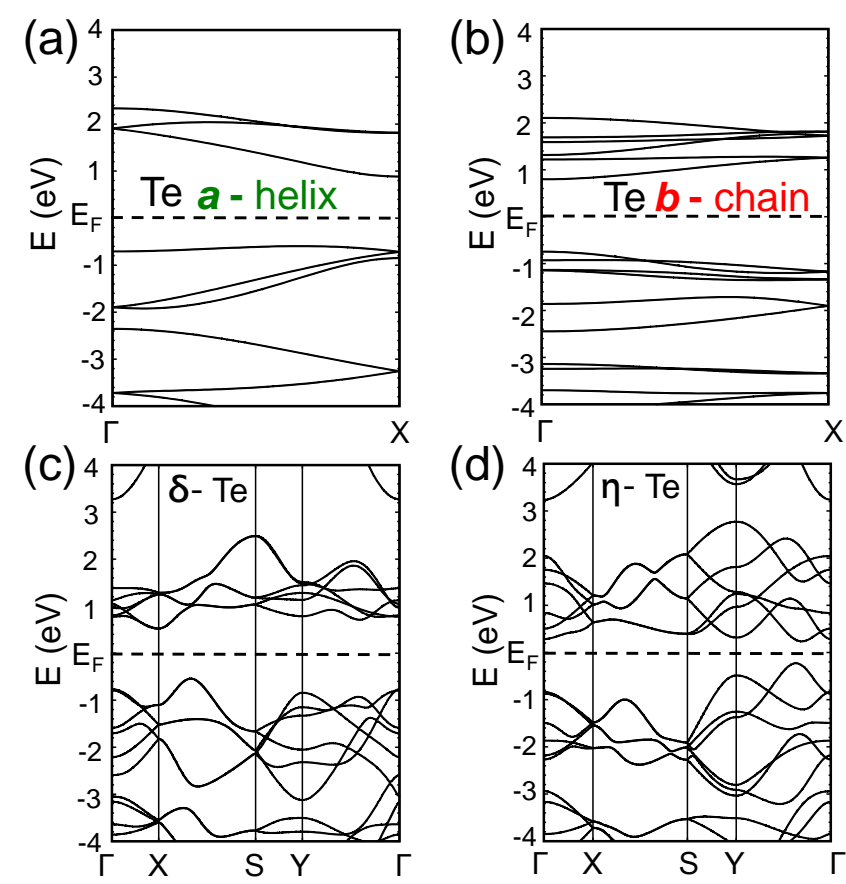

Figure 5: (Color online) Electronic band structure of an isolated (a) $a$-Te helix, (b) $b$-Te chain, isolated (c) $\delta$-Te and (d) $\eta$-Te monolayers obtained using DFT-LDA.

structures are significantly larger than in the 2D layers.

The electronic band structure of different 1D and 2D allotropes of Te is displayed in Fig. 5. We find the trends and main results for the different Te allotropes to be consistent with those for Se, in particular the band gaps in 1D structures to be much larger than in 2D structures. As seen in Fig. 5(a), the band gap of the isolated Te $a$ helix is $1.4 \mathrm{eV}$ wide and indirect. The band gap of the isolated Te $b$ chain, on the other hand, is direct at $\Gamma$ and $1.5 \mathrm{eV}$ wide, as seen in Fig. 5(b). The band structure of $2 \mathrm{D} \delta$-Te, shown in Fig. $5(\mathrm{c})$, has an indirect, $0.9 \mathrm{eV}$ wide band gap. Results for the $2 \mathrm{D}$ allotrope $\eta$-Te, shown in Fig. $5(\mathrm{~d})$, indicate a direct, $0.3 \mathrm{eV}$ wide band gap between $\Gamma$ and $Y$. These numerical results indicate that the LDAbased band gaps in Te are roughly one third of the LDA values found in Se. As mentioned earlier, while DFT-LDA underestimates band gaps, it still provides useful insight into trends in the electronic structure. 


\section{Discussion}

Reported experimental results for 2D chalcogen allotropes include a thin layer of $a$-Se helices on silicon, ${ }^{7}$ a monolayer of $a$-Te helices on graphene,$^{9}$ a substrate-free thin layer of $a$-Te helices, ${ }^{18}$ and $\beta$-Te, a covalently bonded $2 \mathrm{D}$ assembly of $a$ helices. ${ }^{5,6} \mathrm{~A}$ valid question to ask is, why the more stable $2 \mathrm{D} \delta$ allotrope and the $1 \mathrm{D} b$ chain have not been observed. Since interest in group VI elemental 2D structures took off only very recently, it is quite possible that optimum conditions for the synthesis of the proposed allotropes have not been found yet. Established information about 3D chalcogen allotropes consisting of interacting $a$ helices provides only a limited insight into how structures may grow on a $2 \mathrm{D}$ substrate. There, the substrate-chalcogen interaction may play a significant role, such as providing extra stabilization of the more reactive $b$ chains over the $a$ helices.

Without question, the substrate plays a significant role facilitating the $1 \mathrm{D}$ to $2 \mathrm{D}$ transformation in chalcogen structures. Even a weak adsorbate-substrate interaction will confine condensing chalcogen structures in the $2 \mathrm{D}$ space adjacent to the substrate, thus significantly increasing the coalescence rate. To decouple the intrinsic chalcogen reaction energetics from substrate-chalcogen interaction on a particular substrate, we performed all our calculations in vacuum. Specific substrates can be selected that may change the relative stability order in adsorbed chalcogen structures in comparison to such structures in vacuum.

As mentioned before, locating a transformation path in configurational space between very dissimilar structures $a$ and $\delta$, with activation barriers not exceeding $\approx 0.3 \mathrm{eV}$, is a nontrivial task. So far, state-of-the-art global structural searching techniques were unable to locate such a path or other structures that were very different from the native $a$ helices. ${ }^{5}$ We feel that for the time being, understanding the physical origin of strong bonds in terms of $d, \theta, \psi$ and locating a pathway along which only the least energy sensitive structural parameter is modified is a more promising approach to understanding the reaction energy. We located such a path from
$A$ to $D$ that involves only a sequence of changes in $\psi$ within a 9 -atom segment of the chain.

Maybe the most important lesson to learn was that - at least in the class of structures discussed here - releasing structural constraints and increasing the number of degrees of freedom may significantly lower the activation barriers for structural transformations. Even though the initially considered artificial compression of a 2D assembly of $a$ helices with 3 atoms per unit cell to a completely flat structure with 6 degrees of freedom per cell did eventually yield the stable $\delta$ allotrope, the energy invested was unphysically high. Allowing for concerted atomic motion in a 9-atom segment with 27 degrees of freedom lowered the activation barriers significantly. In a related scenario of structural phase transitions in monochalcogenindes, artificial spatial constraints ${ }^{19}$ were also found to significantly affect the energy barriers and thus the critical temperature. ${ }^{20}$ The same behavior can be expected for a wide range of systems undergoing structural phase changes.

\section{Summary and Conclusions}

In summary, based on DFT calculations, we have uncovered the microscopic mechanism of the recently observed structural transition in elemental chalcogens Se and Te from their native bulk structure consisting of $a$ helices to atomically thin 2D layers. We found that the $a$ helices convert to more stable, previously unknown $b$ chains in a multi-step process that involves a point-dislocation motion along the helix. In a zipper-like motion, the $b$ chain reconnects to a related, previously unknown and unusually stable 2D $\delta$ structure of Se and Te. The 1D $a$ helix to $2 \mathrm{D} \delta$ conversion is mildly exothermic with $-0.17 \mathrm{eV} /$ atom for Se and $-0.23 \mathrm{eV} /$ atom for Te. The high structural flexibility allows the helix to exploit many degrees of freedom and thus significantly lower the activation barriers along the complex reaction path to $\lesssim 0.3 \mathrm{eV}$, indicating that the conversion may occur at moderate temperatures. In view of the similar stability of the structurally related $\beta, \delta$ and $\eta$ structures, we expect that all these and maybe even other 
allotropes should be formed at elevated temperatures. We found all $1 \mathrm{D}$ and $2 \mathrm{D}$ chalcogen structures to be semiconducting.

\section{Computational Techniques}

Our calculations of the stability, equilibrium structure, the pathway and dynamics of structural transformations have been performed using density functional theory (DFT) as implemented in the SIESTA ${ }^{21}$ and VASP VI,23 $^{22,}$ codes. Periodic boundary conditions have been used throughout the study, with monolayers represented by a periodic array of slabs separated by a $30 \AA$ thick vacuum region. We compared results using both the Local Density Approximation (LDA) ${ }^{24,25}$ and the Perdew-BurkeErnzerhof $(\mathrm{PBE})^{26}$ exchange-correlation functionals, since LDA typically overbinds and PBE underbinds. We also checked the importance of van der Waals corrections to the total energy by using the optB86b exchange-correlation functional ${ }^{27,28}$ for selected structures. The SIESTA calculations used norm-conserving Troullier-Martins pseudopotentials, ${ }^{29}$ a double$\zeta$ basis including polarization orbitals, and a mesh cutoff energy of 180 Ry to determine the self-consistent charge density, which provided us with a precision in total energy of $\lesssim 2 \mathrm{meV} /$ atom. The VASP calculations were performed using the projector augmented wave (PAW) method ${ }^{23}$ and $500 \mathrm{eV}$ as energy cutoff. The reciprocal space has been sampled by a fine grid $^{30}$ of $10 \times 10 k$-points in the $2 \mathrm{D}$ Brillouin zones (BZ) of the primitive unit cells of the $\delta$ and $\eta$ structures containing 6 atoms each, and $10 k$-points in the $\mathrm{BZ}$ of $1 \mathrm{D} a$ and $b$ chains with 3- and 6-atom unit cells, respectively. Geometries have been optimized using the conjugate gradient (CG) method, ${ }^{31}$ until none of the residual Hellmann-Feynman forces exceeded $10^{-2} \mathrm{eV} / \AA$. Microcanonical and canonical MD calculations were performed using $1 \mathrm{fs}$ time steps. Electronic structure has been calculated using the GW quasiparticle approach ${ }^{32}$ as implemented in the BERKELEYGW package ${ }^{33}$ interfaced with QUANTUMESPRESSO. ${ }^{34}$ In a periodic arrangement, 1D and 2D structures were separated by $17 \AA$ wide vacuum regions. The Brillouin zone of quasi-1D structures was sampled by $36 \times 1 \times 1 \mathrm{k}$-points and that of quasi$2 \mathrm{D}$ structures by $6 \times 14 \times 1 \mathrm{k}$-points. We used 10 Ry as energy cutoff for the plane wave expansion of the dielectric matrix. The quasiparticle energies have been determined by considering the lowest 220 unoccupied conduction bands and accounting for all higher-lying bands using the modified static-remainder approximation. ${ }^{35}$

\section{Supporting Information Avail- able}

The following files are available free of charge. Detailed information regarding the dynamical stability and structural transformations in 1D and 2D Se allotropes. Discussed are the transformation from the 1D $a$ helix to the 2D $\delta$ allotrope by artificial confinement, microscopic transformation from the $\delta$ to the $\eta$ structure, and the microscopic transformation mechanism from the $a$ helix to the $b$ chain. Also provided are video files of MD simulations of a 1D $b$ chain, a 2D $\delta$-Se allotrope, and the transformation from the 1D $a$ helix to the $b$ chain.

\section{Author Information}

\section{Corresponding Author}

${ }^{*}$ E-mail: tomanek@pa.msu.edu

\section{Notes}

The authors declare no competing financial interest.

Acknowledgement D.L. and D.T. acknowledge financial support by the NSF/AFOSR EFRI 2-DARE grant number EFMA-1433459. Computational resources have been provided by the Michigan State University High Performance Computing Center. X.L. acknowledges support by the China Scholarship Council.

\section{References}

(1) Li, L.; Yu, Y.; Ye, G. J.; Ge, Q.; Ou, X.; Wu, H.; Feng, D.; Chen, X. H.; Zhang, Y. Nature Nanotech. 2014, 9, 373-377. 
(2) Liu, H.; Neal, A. T.; Zhu, Z.; Luo, Z.; $\mathrm{Xu}, \mathrm{X}$.; Tomanek, D.; Ye, P. D. ACS Nano 2014, 8, 4033-4041.

(3) Osters, O.; Nilges, T.; Bachhuber, F.; Pielnhofer, F.; Weihrich, R.; Schöneich, M.; Schmidt, P. Angew. Chem. Int. Ed. 2012, 51, 2994-2997.

(4) Pumera, M.; Sofer, Z. Advanced Materials 2017, 29, 1605299.

(5) Zhu, Z.; Cai, X.; Yi, S.; Chen, J.; Dai, Y.; Niu, C.; Guo, Z.; Xie, M.; Liu, F.; Cho, J.H.; Jia, Y.; Zhang, Z. Phys. Rev. Lett. 2017, 119, 106101.

(6) Chen, J.; Dai, Y.; Ma, Y.; Dai, X.; Ho, W.; Xie, M. Nanoscale 2017, 9, 15945-15948.

(7) Qin, J.; Qiu, G.; Jian, J.; Zhou, H.; Yang, L.; Charnas, A.; Zemlyanov, D. Y.; $\mathrm{Xu}$, C.-Y.; Xu, X.; Wu, W.; Wang, H.; Ye, P. D. ACS Nano 2017, 11, 10222 10229.

(8) Greenwood, N. N.; Earnshaw, A. Chemistry of the Elements, 2nd ed.; Butterworth-Heinemann, 1997; pp 645662.

(9) Huang, X.; Guan, J.; Lin, Z.; Liu, B.; Xing, S.; Wang, W.; Guo, J. Nano Lett. 2017, 17, 4619-4623.

(10) Liu, D.; Every, A. G.; Tománek, D. Phys. Rev. B 2016, 94, 165432.

(11) Brown, P. J.; Forsyth, J. B. Acta Crystallogr. A 1996, 52, 408-412.

(12) Cherin, P.; Unger, P. Acta Crystallogr. 1967, 23, 670-671.

(13) Burbank, R. D. Acta Crystallogr. 1951, 4, 140-148.

(14) Adenis, C.; Langer, V.; Lindqvist, O. Acta Crystallogr. C 1989, 45, 941-942.

(15) Jamieson, J. C.; Mcwhan, D. B. J. Chem. Phys. 1965, 43, 1149-1152.
(16) Andharia, E.; Kaloni, T. P.; Salamo, G. J.; Yu, S.-Q.; Churchill, H. O.; BarrazaLopez, S. 2017, preprint, http://arxiv. org/abs/1709.04575.

(17) Tuttle, B.; Alhassan, S.; Pantelides, S. Nanomaterials 2017, 7, 115.

(18) Du, Y.; Qiu, G.; Wang, Y.; Si, M.; Xu, X.; Wu, W.; Ye, P. D. Nano Lett. 2017, 17, 3965-3973.

(19) Fei, R.; Kang, W.; Yang, L. Phys. Rev. Lett. 2016, 117, 097601.

(20) Mehboudi, M.; Fregoso, B. M.; Yang, Y.; Zhu, W.; van der Zande, A.; Ferrer, J.; Bellaiche, L.; Kumar, P.; BarrazaLopez, S. Phys. Rev. Lett. 2016, 117, 246802.

(21) Artacho, E.; Anglada, E.; Dieguez, O.; Gale, J. D.; Garcia, A.; Junquera, J.; Martin, R. M.; Ordejon, P.; Pruneda, J. M.; Sanchez-Portal, D.; Soler, J. M. J. Phys. Cond. Mat. 2008, 20, 064208.

(22) Kresse, G.; Furthmüller, J. Phys. Rev. B 1996, 54, 11169-11186.

(23) Kresse, G.; Joubert, D. Phys. Rev. B 1999, 59, 1758-1775.

(24) Ceperley, D. M.; Alder, B. J. Phys. Rev. Lett. 1980, 45, 566-569.

(25) Perdew, J. P.; Zunger, A. Phys. Rev. B 1981, 23, 5048-5079.

(26) Perdew, J. P.; Burke, K.; Ernzerhof, M. Phys. Rev. Lett. 1996, 7r, 3865-3868.

(27) Klimeš, J.; Bowler, D. R.; Michaelides, A. J. Phys.: Cond. Matt. 2010, 22, 022201.

(28) Klimeš, J.; Bowler, D. R.; Michaelides, A. Phys. Rev. B 2011, 83, 195131.

(29) Troullier, N.; Martins, J. L. Phys. Rev. B 1991, 43, 1993-2006.

(30) Monkhorst, H. J.; Pack, J. D. Phys. Rev. B 1976, 13, 5188-5192. 
(31) Hestenes, M. R.; Stiefel, E. J. Res. Natl. Bur. Stand. 1952, 49, 409-436.

(32) Hybertsen, M. S.; Louie, S. G. Phys. Rev. $B$ 1986, 34, 5390-5413.

(33) Deslippe, J.; Samsonidze, G.; Strubbe, D. A.; Jain, M.; Cohen, M. L.; Louie, S. G. Comp. Phys. Commun. 2012, 183, 1269-1289.

(34) Giannozzi, P.; Baroni, S.; Bonini, N.; Calandra, M.; Car, R.; Cavazzoni, C.; Ceresoli, D.; Chiarotti, G. L.; Cococcioni, M.; Dabo, I.; Corso, A. D.; de Gironcoli, S.; Fabris, S.; Fratesi, G.; Gebauer, R.; Gerstmann, U.; Gougoussis, C.; Kokalj, A.; Lazzeri, M.; MartinSamos, L.; Marzari, N.; Mauri, F.; Mazzarello, R.; Paolini, S.; Pasquarello, A.; Paulatto, L.; Sbraccia, C.; Scandolo, S.; Sclauzero, G.; Seitsonen, A. P.; Smogunov, A.; Umari, P.; Wentzcovitch, R. M. J. Phys.: Cond. Matter 2009, 21, 395502.

(35) Deslippe, J.; Samsonidze, G.; Jain, M.; Cohen, M. L.; Louie, S. G. Phys. Rev. B 2013, 87, 165124. 


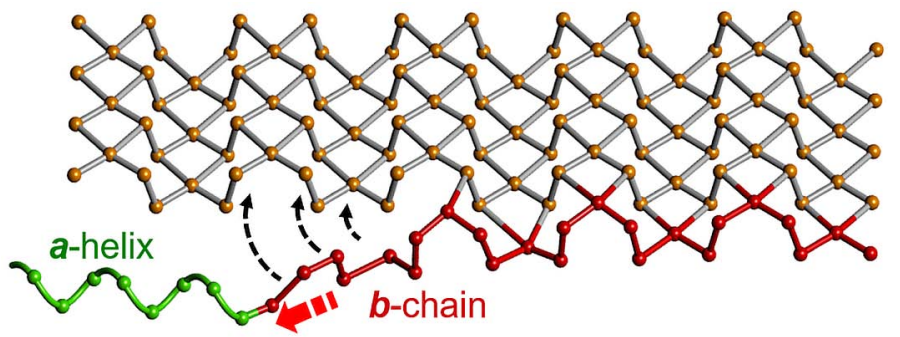

